\title{
EVALUACIÓN DE LA HABILIDAD DIGITAL DE LOS ESTUDIANTES UNIVERSITARIOS: ESTADO DE INGRESO Y POTENCIAL EDUCATIVO.
}

\section{ASSESSMENT OF UNIVERSITY STUDENTS' DIGITAL SKILLS: INITIAL STATUS AND EDUCATIONAL POTENTIAL.}

\author{
Patricia Avitia Carlos; patricia avitia@uabc.edu.mx \\ Irma Uriarte Ramírez; irma ur@uabc.edu.mx \\ Universidad Autónoma de Baja California \\ México
}

\section{RESUMEN}

Con la finalidad de establecer las habilidades digitales de los estudiantes de nuevo ingreso a programas de ingeniería se realizó una investigación descriptiva de corte cuantitativo basada en la autopercepción del estudiante. Se aplicó en línea un cuestionario fundamentado en la Matriz de Habilidades Digitales propuesta por la Universidad Nacional Autónoma de México (UNAM). La muestra se compuso de 114 individuos, cuyos resultados indican la existencia de un nivel medio en competencias en la mayoría de las dimensiones evaluadas. Los resultados contrastan con los usos académicos, lo que da pie a la discusión sobre la necesidad de incorporar aspectos pedagógicos que establezcan un puente entre las competencias tecnológicas y el aprendizaje en medios virtuales.

PALABRAS CLAVE: Competencias Digitales, Habilidades Digitales, Autopercepción, Evaluación.

\begin{abstract}
In order to establish the digital skills of new students to engineering programs, a descriptive quantitative research was conducted based on the student's self-perception. A questionnaire based on the Matrix of Digital Abilities proposed by the National Autonomous University of Mexico (UNAM) was applied online. The sample consisted of 114 individuals, whose results indicate the existence of an average competence level in most of the evaluated dimensions. The results contrast with the academic uses, giving rise to a discussion about the need to incorporate pedagogical aspects capable of establish a bridge between the technological competences and the learning in virtual means.
\end{abstract}

KEYWORDS: Digital Competence, Digital skills, Self-perception, Assessment 


\section{INTRODUCCIÓN}

Existe un reconocimiento entre los principales organismos internacionales promotores de la educación y el desarrollo social sobre la importancia de proveer de competencias digitales a los ciudadanos con la finalidad de integrarlos eficazmente en la sociedad del conocimiento (UNESCO, 2005). Esta postura ha migrado en las últimas décadas a las políticas educativas de diversos países, aunada al reconocimiento estratégico del empleo de Tecnologías de la Información y Comunicación (TIC) en programas de educación superior ya sea a través de modalidades híbridas o virtuales (Artopoulus \& Kozak, 2015) (Bauer \& Catuara Solarz, 2014).

En el caso de la enseñanza de la Ingeniería, existen áreas de oportunidad para el empleo de TIC asociadas al ingreso incrementado por las políticas de estímulo a la matricula en programas técnicos y científicos (Presidencia de la República, 2013). La innovación pedagógica, la actualización del currículo que dé cabida a perfiles profesionales pertinentes y la necesaria optimización en el empleo de la infraestructura de las instituciones educativas han centrado en la formación inicial de estos programas el interés en uso de modalidades de enseñanza apoyadas en TIC.

En años recientes nos hemos familiarizado con expresiones tales como nativos digitales (Prensky M. , 2001) e hiperconectados (Quan-Haase \& Wellman, 2005) mismas que hacen referencia a los miembros de las nuevas generaciones, quienes conviven con y a través de las TIC. Como nos dicen Cabero y Llorente citando a Gordo (2008), se asume la existencia de una familiaridad y dominio en el uso de éstas tecnologías por parte de los jóvenes. Ésta se presenta en el discurso como una ventaja que conllevará su natural integración en una sociedad digital y suscitará la evolución de la educación formal a modalidades no presenciales como alternativa a la educación tradicional en el aula.

De acuerdo con lo anterior, se esperaría que una vez superada la barrera de la accesibilidad los estudiantes universitarios fuesen capaces de realizar actividades de aprendizaje en línea, las cuales pueden incluir desde el ingreso a la plataforma de aprendizaje hasta el desarrollo de evidencias académicas digitales, pasando por la colaboración virtual. Sin embargo, en la práctica no es tan claro que los estudiantes cuenten con el inventario de competencias requeridas para una exitosa incorporación de las TIC al proceso de enseñanza-aprendizaje (Gisbert \& Esteve, 2011). Se parte entonces de determinar, ¿cuáles son estas competencias necesarias? ¿En qué medida las poseen los estudiantes que se incorporan actualmente a la educación superior? Para responder a la primera pregunta hacemos una aproximación al concepto de competencia digital y a las habilidades asociadas que han sido propuestas por distintos organismos internacionales como fundamentales para el ingreso a la sociedad del conocimiento (Ala-Mutka, 2011).

Para dar respuesta al segundo interrogante se realizó un estudio descriptivo de corte cuantitativo para establecer las habilidades digitales con que ingresan los estudiantes a sus estudios de Ingeniería. Se reconoce la existencia de diferencias contextuales correspondientes a la ubicación del estudio por lo que se busca evitar una generalización basada en la importación de referentes. El conocimiento puntual del catálogo de habilidades con que están llegando los 
estudiantes de nuevo ingreso nos permite tanto establecer una asociación de colaboración con el nivel educativo previo, como plantear escenarios adecuados para su adaptación a las modalidades de enseñanza propuestas en el medio universitario.

\subsection{Hacia una definición de la competencia digital.}

Entre la diversidad de términos que han surgido con el advenimiento de las TIC en la educación se encuentra el de competencias digitales. El término emerge a la luz de la sociedad del conocimiento junto a otros conceptos como alfabetización y brecha digital. Ha sido incorporado en documentos que describen políticas oficiales sobre educación, reconociéndose su importancia en la construcción de una sociedad incluyente en la que sus miembros participen de los beneficios del desarrollo de los sistemas de producción de bienes y servicios; puedan contar con oportunidades equitativas para el trabajo, estudio y en general para la mejora de su condición social.

Las competencias digitales son también referidas algunas veces como competencias informacionales, alfabetismo digital, competencia tecnológica, competencias TIC y como la combinación competencias digitales-informacionales, entre otras. Esta multiplicidad de términos obedece a los diferentes contextos en que se emplean, variando el foco de interés y suscitando incertidumbre sobre su identificación. En el presente trabajo empleamos el concepto de habilidad digital dado que consideramos que enfatiza con certidumbre el componente de nuestro interés dentro de la noción más compleja e incluyente de competencia, como es discutido a continuación.

La definición de competencia proporcionada por el Centro de Información sobre Recursos Educativos la encontramos citada por Campos (2016, p. 4), de acuerdo a la cual ésta es definida como la capacidad demostrada de una persona para llevar a cabo una tarea; es decir, la posesión de conocimientos, habilidades y características personales necesarias para satisfacer las exigencias o requisitos especiales de una situación particular.

Por su parte, el concepto de competencia digital se considera actualmente en evolución de la misma manera en que lo están la tecnología de las TIC y sus modalidades de empleo en la educación, el trabajo y la sociedad en general. De acuerdo con llomäki (2014) estamos ante un concepto emergente, cuya delimitación se encuentra abierta, en evolución y que posee por tanto plasticidad. Este autor realiza una extensa revisión a la literatura reciente sobre competencias digitales en políticas oficiales y artículos de investigación educativa, a partir de la cual proponen el uso de una definición adaptable y flexible que sea capaz de integrar los avances tecnológicos y las competencias asociadas que estos demandarán. Se advierte entonces que la necesidad del empleo de recursos transdisciplinares para su abordaje y una ampliación de la visión más allá del campo educativo.

Como resultado de lo anterior nos propone una definición de competencia digital consistente en "las habilidades y prácticas requeridas para el uso de nueva tecnología de manera significativa y como herramienta de aprendizaje, trabajo y tiempo libre, comprendiendo el fenómeno esencial de tecnologías digitales tanto en la sociedad como en la vida propia, y la motivación a participar en el mundo digital como actor activo y responsable" (2014, p. 17). 
Consistentemente, Área (2012) considera que el desarrollo de competencias digitales para la formación de ciudadanos del siglo XXI debe establecerse como un proceso de aprendizaje individual o grupal, donde cada estudiante pueda construirle a través del uso de distintos medios y TICs. De acuerdo con esta visión la tecnología se constituye por sí misma como un espacio dentro del cual los estudiantes aprendan a resolver situaciones problemáticas. Así, el desarrollo de competencias digitales rebasa la habilidad para el empleo de la tecnología y se focaliza en el uso que se hace de la información y comunicación.

En referencia a la Unión Europea (2010), llomäki señala que se considera que junto a las habilidades TIC básicas existen otros aspectos que han recibido menor atención, como lo son el pensamiento crítico en el uso de nuevas tecnologías y medios, uso seguro y responsable, conciencia de riesgos y consideraciones éticas y legales. Tomando esto en consideración se ha seleccionado en el presente estudio la Matriz de habilidades digitales propuesta por UNAM (DGTIC, 2014, p. 5), la cual incluye habilidades asociadas a dichos aspectos. Su propuesta se basa en el entendimiento de las habilidades digitales como el conjunto de saberes relacionados con el uso de herramientas de comunicación, acceso, procesamiento y producción de la información.

En su estudio sobre las habilidades digitales existentes en estudiantes de dos universidades mexicanas, Organista (2016, p. 47) define a éstas como el nivel de dominio que posee el individuo para manejarlas Tecnologías de la Información y la Comunicación (TIC), la cual requiere de la práctica repetitiva de diversas tareas relacionadas con ejecuciones de tipo operativo e informacional.

Las evaluaciones sobre competencias al egreso de bachillerato más extendidas actualmente en México y cuyos resultados son empleados por instituciones nacionales de educación superior como parte de sus procesos de ingreso, son las realizadas por el Centro Nacional de Evaluación para la Educación Superior (CENEVAL). El Examen General de Ingreso a Licenciatura (EXANI-II Admisión) mide las competencias genéricas del estudiante que desea ingresar a un programa de licenciatura mientras que tanto el EXANI II Diagnóstico como el DOMINA-CDE de egreso de educación media superior hacen lo propio con las competencias disciplinares extendidas (CENEVAL, 2016) (CENEVAL, 2015) . Sin embargo, ni el EXANI II 2016 en su versión Admisión ni él examen DOMINA evalúan las competencias digitales. Una aproximación a la evaluación de las mismas aparece en el EXANI II Diagnóstico para el módulo de Arquitectura, Urbanismo y Diseño el cual cuenta con el área disciplinar de Tecnologías de la Información y Comunicación. Consideramos que de manera adicional a la identificación de habilidades digitales esperadas es conveniente una estandarización y uso de las métricas con fines de diagnóstico.

\subsection{La evaluación de las competencias digitales.}

Pueden identificarse estudios previos orientados a determinar el nivel real de competencia digital de los estudiantes, así como para conocer los usos que hacen de las TIC tanto dentro de sus actividades de estudio como en aquellas de ocio. Estos estudios buscan proporcionar una base sólida para el desarrollo de políticas y programas de alfabetización digital que correspondan a un diagnóstico local. En varios casos se encuentran basados en la 
autopercepción de los estudiantes con relación a su nivel de competencia con enfoque en la habilidad técnica para el empleo. Al contrastar los resultados obtenidos se puede observar la homogeneidad en cuanto al desarrollo percibido de habilidades digitales, no así en lo que concierne al aprovechamiento de las mismas con fines formativos en el aula.

En el estudio sobre competencia digital informacional autopercibida que realizan en el programa de Formación de Profesores de una Universidad española, Pech (2016) evalúa las dimensiones de Competencia Técnica (AT), Pedagógica (AP), Social (AS), de Gestión y Organización (AGE), de Comunicación con TIC (AC), de Formación Profesional (ADP) y de Aplicación TIC en la Universidad (AUS). Encuentra que estos estudiantes declaran ser competentes en el área tecnológica, digital e informacional. En cuanto a las áreas de Formación Profesional y de Gestión y Organización se autoevalúan como moderadamente competentes.

Por su parte, en un estudio similar realizado por Cabero (2008) con estudiantes españoles de nivel secundario se encuentra que ellos se califican como verdaderamente competentes en el manejo de procesadores de texto y herramientas de comunicación síncrona y asíncrona por Internet (uso instrumental); moderadamente competentes en manejo de información y ligeramente competentes en evaluación y selección de la información. Sin embargo, al ser evaluados por sus docentes en los mismos rubros su calificación promedio es inferior o lo que es lo mismo, los alumnos se autocalifican mejor de lo que lo hacen sus docentes.

Los estudiantes peruanos egresados de secundaria que participaron en el estudio sobre alfabetización digital de Arnao \& Gamonal (2016) consideran mayoritariamente que poseen habilidades informáticas de nivel medio (41\%) o avanzado (31\%). De acuerdo con este estudio, los estudiantes poseen dispositivos de acceso a Internet como laptop, teléfono inteligente o computadora de escritorio y el tiempo de dedicación semanal a su conexión a la red corresponde para la mayoría (86.62\%) a más de 6 horas semanales. El trabajo concluye sin embargo, que didácticamente no se ha aprovechado el potencial de conexión y manejo de herramientas tecnológicas de los estudiantes para dotarlos de mayor capacidad digital lectoraescritora la cual es base para el desarrollo de competencias de investigación y difusión en la sociedad del conocimiento.

En su estudio sobre habilidades digitales de los estudiantes de dos universidades mexicanas, Organista (2016) refiere un alto porcentaje de posesión de equipo de cómputo (93\%) y nivel alto de habilidad digital. No se identifica una relación directa entre ésta capacidad y el desempeño académico pero se reconoce el amplio campo de aplicaciones a su alcance y la ocasión que ello representa para ser explotado académicamente.

\section{METOdOLOGÍA.}

A fin de conocer las habilidades digitales con que cuentan los estudiantes al ingresar a la universidad, se realizó una encuesta autoadministrada a 114 estudiantes de primer semestre de Ingeniería en una universidad pública del Noroeste de México. Para la misma se utilizó un cuestionario con dos secciones: tres preguntas cerradas sobre sus características de acceso y la segunda consistente en 35 preguntas cerradas categorizadas con afirmaciones sobre sus 
habilidades digitales. Para la segunda sección las respuestas se presentaron a través de una escala de Likert de cinco puntos.

El instrumento empleado se basó en la matriz de habilidades digitales propuesta por la Dirección General de Cómputo y de Tecnologías de la Información y Comunicación (DGTIC) de la UNAM (2014). Dicha matriz cubre en total 339 rubros de acuerdo al nivel de habilidad, los cuales varían desde el Nivel 1 o básico hasta el Nivel 3 o avanzado. Adicionalmente, los rubros se encuentran organizados en 8 temas o categorías principales las cuales son: 1) Acceso a la información; 2) Comunicación y colaboración en línea; 3) Seguridad de la información; 4) Procesamiento y administración de la información; 5) Manejo de medios; 6) Características de las computadoras; 7) Ambientes virtuales de aprendizaje; 8) Recursos y herramientas tecnológicas de apoyo a la enseñanza.

Para la integración del cuestionario se redujeron las habilidades encuestadas, centrándolas en aquellas asociadas con el uso que como estudiantes requieren dentro de la educación en línea y en la capacidad de hacer un uso seguro de la tecnología. Se eliminó la categoría 8 sobre Recursos y herramientas tecnológicas de apoyo a la enseñanza por considerarse que evalúa habilidades asociadas a la docencia o aplicaciones específicas de desarrollo de ambientes y aplicaciones a través de la programación. De las siete categorías restantes se eliminaron rubros que podían resultar redundantes o que conseguían darse por satisfechas evaluando el siguiente nivel de habilidad. De igual manera se eliminaron competencias que debían existir para responder al cuestionario como por ejemplo el acceso a la plataforma educativa. Al final se obtuvieron los 30 reactivos empleados y organizados en las siete categorías mostradas en la Tabla

\begin{tabular}{|l|c|}
\hline \multicolumn{1}{|c|}{ Categoría } & Reactivos \\
\hline 1) Acceso a la información & 8 \\
\hline 2) Comunicación y colaboración & 8 \\
\hline 3) Seguridad de la información & 4 \\
\hline 4) Administración de la información & 6 \\
\hline 5) Manejo de medios & 3 \\
\hline 6) Hardware & 2 \\
\hline 7) Ambientes virtuales & 4 \\
\hline
\end{tabular}

Tabla 1. Relación de reactivos empleados por categoría.

Se tomó una muestra de 114 estudiantes de una población de 430 alumnos de nuevo ingreso en el Tronco Común de Ciencias de la Ingeniería, con un intervalo de confianza del $95 \%$ y un margen de error del 7.88\% (Creative Research Systems, 2012). La aplicación del cuestionario se 
realizó dentro del curso de primer semestre "Introducción a la Ingeniería" a través de la plataforma Blackboard y haciendo uso de su herramienta de evaluación "Sondeos".

Los resultados muestran una alta consistencia interna (coeficiente alfa de Cronbach $=0.95$ ). En la Tabla 2 se muestran los coeficientes para las distintas categorías de habilidades indagadas. Se omiten las categorías "Manejo de medios" y "Hardware" debido a que se considera que el coeficiente no es apropiado para una escala que explora un solo factor, siendo tres el número mínimo de ítems (Oviedo \& Campo-Arias, 2005).

\begin{tabular}{|l|l|}
\hline \multicolumn{1}{|c|}{ Categoría } & $\boldsymbol{\alpha}$ \\
\hline 1) Acceso a la información & 0.81 \\
\hline 2) Comunicación y colaboración & 0.84 \\
\hline 3) Seguridad de la información & 0.64 \\
\hline 4) Administración de la información & 0.83 \\
\hline 7) Ambientes virtuales & 0.83 \\
\hline
\end{tabular}

Tabla 2. Coeficiente alfa de Cronbach por dimensión o categoría.

\section{RESULTADOS}

De acuerdo a los resultados recabados, en cuanto a las características de acceso el $65 \%$ de los encuestados indica conectarse diariamente entre tres y cuatro horas al Internet. Realizan habitualmente su conexión desde el hogar (54\%) o desde su dispositivo móvil (12\%). Los dispositivos mayormente empleados por los estudiantes son la computadora portátil (37\%), el teléfono inteligente (25\%) y la computadora de escritorio (22\%).

Para la interpretación de las respuestas obtenidas en la segunda sección, se emplea una escala de intervalo entre 1 y 5 cuyos resultados se observan en la Tabla 3 . El nivel general de habilidades digitales autopercibido por los estudiantes se establece en un valor medio de 3.65.

Así, en la categoría de Acceso a la Información se observa que la mayoría de los estudiantes que respondieron la encuesta cuenta con un nivel 2 o medio de habilidades al considerarse capaces de realizar actividades asociadas a la búsqueda, organización y valoración de la información disponible en la red, no así para emplear búsquedas avanzadas a través de operadores booleanos o metabuscadores.

En cuanto a la Comunicación y Colaboración, existe una amplia participación de los estudiantes en redes sociales, así como una autoeficacia percibida para actividades de colaboración en línea 
mismas que fueron clasificadas como de nivel 2 o medio. De igual manera se consideran competentes para participar en actividades básicas dentro de los ambientes virtuales de aprendizaje.

\begin{tabular}{|c|c|c|c|}
\hline HABILIDAD & NIVEL & MEDIA & D.E. \\
\hline \multicolumn{4}{|l|}{ 1) ACCESO A LA INFORMACIÓN } \\
\hline $\begin{array}{l}\text { Cuando busco información en Internet uso principalmente sitios } \\
\text { institucionales como fuente. }\end{array}$ & Nivel 1 & 3.76 & 0.97 \\
\hline $\begin{array}{l}\text { Soy capaz de realizar trámites administrativos en línea, como solicitud } \\
\text { de constancias, inscripción a seguro facultativo, etc. }\end{array}$ & Nivel 1 & 3.91 & 1.12 \\
\hline Empleo bibliotecas digitales en línea. & Nivel 2 & 3.67 & 1.02 \\
\hline Sé cómo citar en un documento las fuentes de Internet empleadas. & Nivel 2 & 3.84 & 1.10 \\
\hline Organizo mis sitios favoritos de Internet en carpetas. & Nivel 2 & 3.18 & 1.38 \\
\hline Sé cómo personalizar la barra de herramientas del navegador. & Nivel 2 & 3.94 & 1.20 \\
\hline Utilizo operadores booleanos para hacer búsquedas especializadas. & Nivel 3 & 2.81 & 1.01 \\
\hline Sé identificar metabuscadores. & Nivel 3 & 3.02 & 1.21 \\
\hline \multicolumn{4}{|l|}{ 2) COMUNICACIÓN Y COLABORACIÓN } \\
\hline $\begin{array}{l}\text { Publico y comparto archivos (audio, video, imagen y documentos) en } \\
\text { redes sociales (Facebook, Twitter, Pinterest). }\end{array}$ & Nivel 1 & 3.28 & 1.40 \\
\hline Utilizo chats o video chat (Hangouts, Skype, WhatsApp). & Nivel 1 & 4.08 & 1.09 \\
\hline $\begin{array}{l}\text { Participo en grupos (Grupos de Facebook, Grupos de Google, Yahoo } \\
\text { Groups, Comunidad Google +,etc). }\end{array}$ & Nivel 1 & 4.20 & 1.03 \\
\hline Sé crear, abrir y eliminar archivos compartidos. & Nivel 1 & 4.04 & 1.09 \\
\hline Instalo antivirus para Android y Windows en mi dispositivo móvil. & Nivel 2 & 3.64 & 1.29 \\
\hline Gestiono carpetas o etiquetas de correo electrónico. & Nivel 2 & 3.46 & 1.10 \\
\hline Sincronizo dos o más cuentas de correo electrónico. & Nivel 2 & 3.81 & 1.25 \\
\hline Soy capaz de editar, compartir y descargar documentos en la nube. & Nivel 2 & 3.85 & 1.16 \\
\hline
\end{tabular}




\begin{tabular}{|c|c|c|c|}
\hline \multicolumn{4}{|l|}{ 7) AMBIENTES VIRTUALES } \\
\hline $\begin{array}{l}\text { Conozco cómo enviar tareas y revisar los comentarios del docente en la } \\
\text { plataforma educativa. }\end{array}$ & Nivel 2 & 4.37 & 0.93 \\
\hline Puedo localizar, descargar y utilizar recursos y materiales. & Nivel 2 & 4.19 & 0.97 \\
\hline $\begin{array}{l}\text { Puedo participar en wikis dentro de la plataforma educativa de la } \\
\text { institución. }\end{array}$ & Nivel 2 & 3.39 & 0.97 \\
\hline $\begin{array}{l}\text { Sé cómo participar en un foro: colocar un nuevo tema de discusión, } \\
\text { ordenar respuestas. }\end{array}$ & Nivel 2 & 3.86 & 1.09 \\
\hline \multicolumn{4}{|l|}{ 4) ADMINISTRACIÓN DE LA INFORMACIÓN } \\
\hline $\begin{array}{l}\text { Puedo trabajar con documentos en procesadores de texto: crear, abrir, } \\
\text { guardar y editar documentos. }\end{array}$ & Nivel 1 & 4.28 & 0.99 \\
\hline $\begin{array}{l}\text { Puedo trabajar con hojas de cálculo: crear, abrir o editar libros y hojas, } \\
\text { nombrar, editar, insertar, ocultar y mover. }\end{array}$ & Nivel 1 & 3.78 & 1.18 \\
\hline $\begin{array}{l}\text { Me considero eficiente para crear bases de datos con un gestor de } \\
\text { bases de datos. }\end{array}$ & Nivel 2 & 3.03 & 1.12 \\
\hline $\begin{array}{l}\text { Soy capaz de paginar un documento con secciones en un procesador de } \\
\text { texto. }\end{array}$ & Nivel 2 & 3.64 & 1.12 \\
\hline Soy capaz de crear y editar macros en una hoja de cálculo. & Nivel 3 & 3.26 & 1.21 \\
\hline $\begin{array}{l}\text { Sé crear tablas dinámicas de contenido e índices en un procesador de } \\
\text { texto. }\end{array}$ & Nivel 3 & 3.59 & 1.10 \\
\hline \multicolumn{4}{|l|}{ 3) SEGURIDAD DE LA INFORMACIÓN } \\
\hline $\begin{array}{l}\text { Acostumbro cerrar sesiones (redes sociales, correo electrónico, } \\
\text { blackboard), sobre todo en equipos públicos. }\end{array}$ & Nivel 1 & 4.43 & 0.99 \\
\hline Borro historial y cookies al emplear equipo ajeno. & Nivel 2 & 3.77 & 1.24 \\
\hline $\begin{array}{l}\text { Identifico las diferentes formas de robo de datos: spyware, malware, } \\
\text { registro de captura de teclas. }\end{array}$ & Nivel 2 & 3.22 & 1.32 \\
\hline Hago periódicamente respaldos de seguridad de la información. & Nivel 2 & 3.25 & 1.36 \\
\hline
\end{tabular}




\begin{tabular}{|c|c|c|c|}
\hline Cito las fuentes de donde se extraen las imágenes que utilizo. & Nivel 2 & 3.24 & 1.24 \\
\hline $\begin{array}{l}\text { Soy capaz de integrar una secuencia de imágenes para crear una } \\
\text { animación. }\end{array}$ & Nivel 3 & 3.07 & 1.23 \\
\hline Hago uso de software para conversión, creación y edición de audio. & Nivel 3 & 3.25 & 1.26 \\
\hline \multicolumn{4}{|l|}{ 6) HARDWARE } \\
\hline $\begin{array}{l}\text { Sé instalar y configurar dispositivos periféricos inalámbricos y } \\
\text { alámbricos, multifuncionales, impresoras, teclados, cámaras web, } \\
\text { etcétera. }\end{array}$ & Nivel 1 & 3.99 & 1.09 \\
\hline $\begin{array}{l}\text { Me considero eficiente para instalar un sistema operativo en mi equipo } \\
\text { de cómputo. }\end{array}$ & Nivel 3 & 3.60 & 1.23 \\
\hline
\end{tabular}

Tabla 3. Resultados obtenidos en la aplicación del instrumento.

En la Administración de la Información (empleo de procesadores de texto, hojas de cálculo, bases de datos) necesarios para el desarrollo de actividades académicas de procesamiento, existe dispersión en la calificación que se otorgan los estudiantes encontrándose mayor confianza en su manejo de procesadores de texto por encima de las bases de datos.

La Seguridad de la Información es otro tema que ha sido señalado como primordial para el desarrollo de una ciudadanía digital y parte de la competencia digital esperada. En este rubro los estudiantes evaluados se perciben con un nivel medio de habilidad digital estando habituados a realizar la acción básica de seguridad informática de cerrar sesiones y borrar historiales en los equipos de cómputo.

Por su parte en el Manejo de Medios y Hardware existe una capacidad autopercibida de nivel medio, reconociéndose los estudiantes como capaces de instalar hardware o software pero capaces en menor medida de emplear software especializado para edición de imágenes o creación de secuencias de animación.

\section{DISCUSIÓN DE RESULTADOS.}

Con el presente estudio se presenta información contextualizada que posibilita la articulación de los resultados existentes en el egreso de bachillerato con las necesidades de formación para la educación superior en modalidades a distancia. Para ello se evaluó la habilidad digital de acuerdo la matriz propuesta para éste nivel educativo dentro del ámbito del sistema educativo nacional y evadiendo la importación de referentes externos. Se identifican las distintas 
dimensiones propuestas para el desarrollo de una ciudadanía digital global y se tiene obtiene conocimiento del potencial de desarrollo de los estudiantes dada sus características de acceso.

Al comparar los resultados de los estudios conducidos sobre competencias digitales anteriormente citados con el estudio realizado en estudiantes de ingeniería y presentado en este trabajo, se observa que en todos los casos revisados los estudiantes poseen habilidades digitales básicas e intermedias. La existencia de estas habilidades posibilita su acceso y participación en programas académicos en modalidades en línea como viene ya ocurriendo en la mayoría de los casos. Sin embargo, dado que los estudios apuntan también a un conocimiento de tipo instrumental de la tecnología y a una falta de certeza en la valoración y manejo de la información a la que acceden, se percibe tanto la necesidad de establecer programas que doten de dichas competencias a los estudiantes como de desarrollo de parámetros mínimos de desempeño esperado.

Es necesario partir de la realización de evaluaciones de la competencia digital en estudiantes en contextos internacionales y nacionales, los cuales puedan indagar sobre la existencia de las mismas y enfatizar distintas perspectivas de interés como lo son la disminución de la brecha digital, el desarrollo de competencias profesionales o investigativas, etc. Actualmente, los estudios empleados proponen el empleo de distintos instrumentos de evaluación lo cual obstaculiza la comparación y el estudio de evolución de indicadores en distintas regiones y grupos de edad así como su uniforme adaptación a los naturales cambios en la tecnología disponible. Tal y como indican Gesbert \& Esteve (2011) el reto a asumir desde las universidades es necesario evidenciar a través de procesos formativos y de acreditación el nivel de competencia con que los estudiantes llegan a fin de formar mejores profesionales.

\section{REFERENCIAS.}

Ala-Mutka, K. (2011). Mapping digital competence: towards a conceptual understanding. Sevilla: European Comission. http://ftp.jrc.es/EURdoc/JRC67075 TN.pdf

Area, M., \& Guarro, A. (2012). La alfabetización informacional y digital: fundamentos pedagógicos para la enseñanza y el aprendizaje competente. Revista Española de Documentación Científica, 46-74. http://dx.doi.org/10.3989/redc.2012.mono.977

Arnao Vásques, M. O., \& Gamonal Torres, C. E. (2016). Lectura y escritura con recursos TIC en Educación Superior. Evaluación de la competencia digital. INNOEDUCA, 64-73.

Artopoulus, A., \& Kozak, D. (2015, Enero). Tsunami 1:1: estilos de adopción de tecnología en la educación latinoamericana. Revista Iberoamericana de Ciencia, Tecnología y Sociedad, 10(28), 2-32.

Bauer, R., \& Catuara Solarz, M. (2014). Políticas de Infraestructura, Equipamiento y Conectividad. In M. T. Lugo, N. López, \& L. Toranzos, Políticas TIC en los sistemas educativos de Latinoamérica. Informe sobre tendencias sociales y educativas en América Latina 2014. (pp. 71-108). Buenos Aires: UNESCO-OEI. 
Cabero Almenara, J., \& Llorente Cejudo, M. C. (2008). La alfabetización digital de los alumnos. Competencias digitales para el siglo XXI. Revista portuguesa de pedagogía, 7-28.

CENEVAL. (2015). Guía del Examen Domina las Competencias Disciplinares Extendidas. Ciudad de México: Centro Nacional para la Evaluación de la Educación Superior A.C.

CENEVAL. (2016). Guía Exani-II. Ciudad de México: Centro Nacional de Evaluación para la Educación Superior A.C. http://www.ceneval.edu.mx/

Creative Research Systems. (2012). The Survey System. Retrieved 2016, from http://www.surveysystem.com/sscalc.htm\#one

DGTIC, U. (2014, 05 18). Matriz de habilidades digitales. Retrieved 06 2015, from http://www.educatic.unam.mx

Gisbert, M., \& Esteve, F. (2011). Digital learners: la competencia digital de los estudiantes universitarios. La cuestión universitaria, 48-59.

Ilomaki, L., Paavola, S., Lakkala, M., \& Kantosalo, A. (2014). Digital competence - an emergent boundary concept for policy and educational research. Education Information Technology, 1-25.

Organista, J., Sandoval Silva, M., \& McAnally Sallas, L. (2016). Estimación de las habilidades digitales con propósito educativo de estudiantes de dos universidades públicas mexicanas. Revista electrónica de tecnología educativa(57), 46-62.

Oviedo, H. C., \& Campo-Arias, A. (2005). Aproximación al uso del coeficiente alfa de Cronbach. Revista colombiana de psiquiatría, 572-580.

Pech, S. J., \& Prieto, M. E. (2016). La medición de la competencia digital e informacional. In T. De León, J. García, \& E. Orozco, Desarrollo de las competencias para el Siglo XXI (pp. 2650). Miami: Humboldt Group Foundation.

Prensky, M. (2001). Digital natives, digital immigrants. On the horizon, 9(5), 1-6.

Presidencia de la República. (2013). Plan Nacional de Desarrollo 2013-2018. Ciudad de México: Gobierno de la República. http://pnd.gob.mx/

Quan-Haase, A., \& Wellman, B. (2005). Hyperconnected Net Work. In C. Heckscher, \& P. Alder, The corporation as a collaborative community (pp. 281-333). Oxford University Press.

UNESCO. (2005). Hacia las sociedades del conocimiento. Paris: ONU. http://unesdoc.unesco.org/images/0014/001419/141908s.pdf 


\section{Para referenciar este artículo:}

Avitia Carlos, P. \& Uriarte Ramírez, I. (2017). Evaluación de la habilidad digital de los estudiantes universitarios: estado de ingreso y potencial educativo. EDUTEC, Revista electrónica de Tecnología Educativa, 61. Recuperado de: http://dx.doi.org/10.21556/edutec.2018.61 